\title{
Constraints on the opacity profile of the sun from helioseismic observables and solar neutrino flux measurements
}

\author{
F.L. Villante ${ }^{1}$ \\ ${ }^{1}$ Università dell'Aquila and INFN - LNGS, L'Aquila - Italy
}

\begin{abstract}
Motivated by the solar composition problem and by using the recently developed Linear Solar Model approach [1, we analyze the role of opacity and metals in the sun. After a brief discussion of the relation between the effects produced by a variation of composition and those produced by a modification of the radiative opacity, we calculate numerically the opacity kernels that, in a linear approximation, relate an arbitrary opacity variation to the corresponding modification of the solar observable properties. We use these opacity kernels to discuss the present constraints on opacity (and composition) provided by helioseismic and solar neutrino data.
\end{abstract}




\section{Introduction}

In the last few years a new solar problem has emerged. Recent determinations of the photospheric heavy element abundances [2, 3, 4] indicate that the sun metallicity is lower than previously assumed [5]. Solar models that incorporate these lower abundances are no more able to reproduce the helioseismic results. As an example, the sound speed predicted by standard solar models (SSMs) implementing the heavy element admixture of [2] disagrees at the bottom of the convective envelope by $\sim 10 \sigma$ with the value inferred by helioseismic data (see e.g. [6] and black dashed line in fig.1). In addition, the predicted surface helium abundance is lower by $\sim 6 \sigma$ and the radius of the convective envelope is larger by $\sim 15 \sigma$ with respect to the helioseismic results. Detailed studies have been done to resolve this controversy, see e.g. [7]. The latest determinations of the solar photospheric composition [3, 4] alleviate the discrepancies but a definitive solution of the "solar composition problem" still has to be obtained.

The main effect of changing the heavy element admixture is to modify the opacity profile of the sun. It is, thus, evident that the comprehension of the solar composition problem is intimately related to understanding the role of opacity in solar modelling. Several authors have investigated the effects of opacity changes on the solar structure by using different methods and assumptions (see e.g. [8] and references therein). Here, we continue their work, completing and extending the analysis of [8] by using a different and original approach. The final goal is to provide the instruments to analyze in transparent and efficient way the role of the opacity in the sun and to perform a critical "step-by-step" discussion of the present constraints on opacity (and composition) provided by observable properties of the sun.

In order to calculate the effects of arbitrary opacity changes on the sun, we use the linear solar model (LSM) approach, presented in 1, 9] and briefly summarized in the appendix. In this approach, the structure equations of the present sun are linearized and, by estimating the (variation of) the present solar composition from the (variation of) the nuclear reaction rates and elemental diffusion efficiency in the present sun, we obtain a linear system of ordinary differential equations that can be easily solved and that completely determines the physical and chemical properties of the sun. It was shown in [1] that this kind of approach reproduces with good accuracy the results of non-linear evolutionary solar models and, thus, can be used to study the role of parameters and assumptions in solar model construction.

By considering localised opacity changes in LSM approach, we determine numerically the kernels that, in a linear approximation, relate an arbitrary opacity variation to the corresponding modification of the solar observable properties. These opacity kernels are useful in several respects. First, they allow us to individuate the region of the sun whose opacity is probed with maximal sensitivity by each observable quantity. Then, they permit us to show that effects produced by variations of opacity in different region of the sun can compensate among each others. Finally, they will be used to discuss how the different pieces of observational information cooperate to determine the present constraints on the opacity profile of the sun.

The plan of the paper is the following. In sect. 2 , we discuss the relation between the effects produced by a variation of the heavy element admixture and those produced by a modification of the radiative opacity. We show that the relevant quantity is the variation of the opacity profile $\delta \kappa(r)$ defined in eq. (2), that is approximately given by the superposition of the intrinsic opacity change $\delta \kappa_{\mathrm{I}}(r)$ and the composition opacity change $\delta \kappa_{Z}(r)$, defined in eqs. (3) and (5) respectively. In sect. 3, we define the opacity kernels and we describe the method adopted to calculate them. In sect. 4, we calculate numerically the kernels for the squared isothermal sound speed $u(r) \equiv P(r) / \rho(r)$, the surface helium abundance $Y_{\mathrm{b}}$, the radius of the convective envelope $R_{\mathrm{b}}$ and the various neutrino fluxes $\Phi_{\nu}$. Moreover, we discuss the constraints on $\delta \kappa(r)$ provided by the present observational data. Finally, in sect. 5 we summarize our results. A conclusive view of the constraints on $\delta \kappa(r)$ is provided by fig. 8 , 

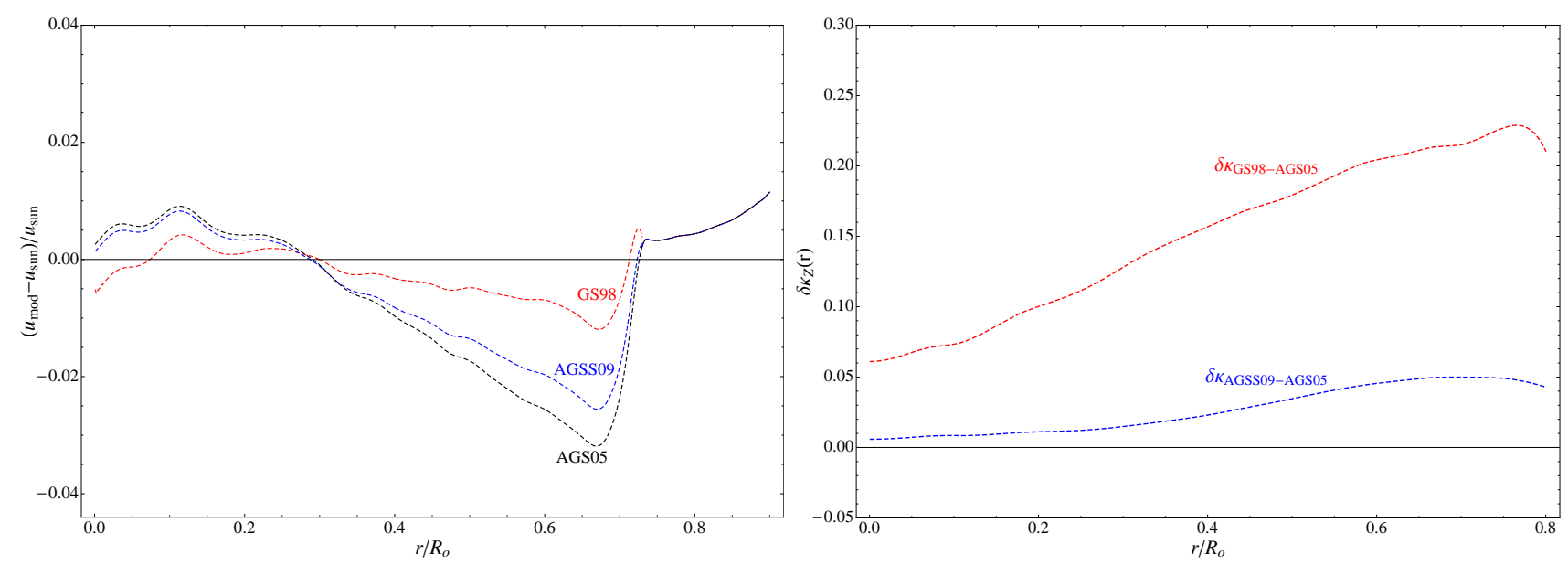

Figure 1: Left Panel: The difference between the squared isothermal sound speed inferred from helioseismic data and the predictions of solar models implementing AGS05 (black), GS98 (red) and AGSS09 (blue) heavy element admixtures. Right panel: The composition opacity changes $\delta \kappa_{Z}(r)$ that corresponds to using the GS98 (red) and the AGSS09 (blue) composition in place of the AGS05 composition.

\section{The relation between opacity and metals}

We consider a modification of the opacity $\kappa\left(\rho, T, Y, Z_{\mathrm{i}}\right)$ and/or of the heavy element photospheric admixture $\left\{z_{\mathrm{i}}\right\}$, expressed here in terms of the quantities $z_{\mathrm{i}} \equiv Z_{\mathrm{i}, \mathrm{b}} / X_{\mathrm{b}}$ where $Z_{\mathrm{i}, \mathrm{b}}$ is the surface abundance of the $i$-element and $X_{\mathrm{b}}$ is that of hydrogen. If we neglect the role of metals in the equation of state and in the energy generation coefficient, the only effect of these changes is to modify radiative energy transport in the sun. The relevant parameter, in this respect, is the total variation of the opacity in the shell $r$ of the present sun, given by:

$$
\delta \kappa^{\mathrm{tot}}(r)=\frac{\kappa\left(\rho(r), T(r), Y(r), Z_{\mathrm{i}}(r)\right)}{\bar{\kappa}\left(\bar{\rho}(r), \bar{T}(r), \bar{Y}(r), \bar{Z}_{\mathrm{i}}(r)\right)}-1
$$

where $\bar{\kappa}\left(\bar{\rho}(r), \bar{T}(r), \bar{Y}(r), \bar{Z}_{\mathrm{i}}(r)\right)$ is the opacity profile of the SSM, while $\kappa\left(\rho(r), T(r), Y(r), Z_{\mathrm{i}}(r)\right)$ is the opacity profile of the solar model that implements the modified opacity and photospheric composition 1 . We note that the quantity $\delta \kappa^{\text {tot }}(r)$ is not related in a direct way to the performed variations of opacity and composition. In order to calculate it, we have to take into account that the "perturbed" sun has different density $(\rho)$, temperature $(T)$ and chemical composition profiles ( $Y$ and $Z_{\mathrm{i}}$ ) with respect to the SSM. These are not known a priori but have to be obtained as a result of numerical solar modelling.

A relevant simplification is obtained in the LSM approach presented in ref. [1], where one assumes that: $i$ ) the performed changes of opacity and heavy element admixture are small; ii) the (variation of) the chemical composition of the sun can be estimated from the (variation of) nuclear reaction rate and diffusion efficiency of the present sun; iii) the variation of the metal admixture has a negligible direct effect on nuclear production of helium and on diffusion efficiency. In this case, the relation between $\delta \kappa^{\text {tot }}(r)$ and the performed variation of opacity and admixture can be worked out explicitly, as it is described in the appendix. It can be shown, moreover, that the source term $\delta \kappa(r)$ that drives the modification of the solar properties $\mathrm{S}^{2}$ and that can be constrained by observational data can be written

\footnotetext{
${ }^{1}$ The notation $\bar{Q}$ indicates, here and in the following, the SSM's value for the generic quantity $\mathrm{Q}$.

${ }^{2}$ In mathematical terms, the quantity $\delta \kappa(r)$ represents the inhomogeneous term in the linearized structure equation of
} 


\begin{tabular}{l|cc|ccccc} 
& $\delta R_{\mathrm{b}}$ & $\Delta Y_{\mathrm{b}}$ & $\delta \Phi_{\mathrm{pp}}$ & $\delta \Phi_{\mathrm{Be}}$ & $\delta \Phi_{\mathrm{B}}$ & $\delta \Phi_{\mathrm{O}}$ & $\delta \Phi_{\mathrm{N}}$ \\
\hline \hline GS98 - AGS05 & -0.019 & 0.019 & -0.011 & 0.14 & 0.26 & 0.56 & 0.49 \\
AGSS09 - AGS05 & -0.0056 & 0.0028 & -0.0014 & 0.018 & 0.034 & 0.13 & 0.12 \\
\hline
\end{tabular}

Table 1: The variations of helioseismic and solar neutrino observables produced by a variation of the heavy element admixture. The above results have been estimated within the LSM approach, by applying the opacity changes $\delta \kappa_{\mathrm{Z}}(r)$ shown in the right panel of fig 1 . For CNO neutrinos, we also considered that the fluxes scales proportionally to the total CN-abundance. Note that the absolute variation is reported for the surface helium abundance, whereas the relative variations are shown for all the other quantities.

as the sum of two contributions:

$$
\delta \kappa(r)=\delta \kappa_{\mathrm{I}}(r)+\delta \kappa_{\mathrm{Z}}(r)
$$

The first term $\delta \kappa_{\mathrm{I}}(r)$, which we refer to as intrinsic opacity change, represents the fractional variation of the opacity along the SSM profile and it is given by:

$$
\delta \kappa_{\mathrm{I}}(r)=\frac{\kappa\left(\bar{\rho}(r), \bar{T}(r), \bar{Y}(r), \bar{Z}_{i}(r)\right)}{\bar{\kappa}\left(\bar{\rho}(r), \bar{T}(r), \bar{Y}(r), \bar{Z}_{i}(r)\right)}-1
$$

This contribution is obtained when we revise the opacity function $\kappa\left(\rho, T, Y, Z_{\mathrm{i}}\right)$ and/or we introduce new effects, like e.g. the accumulation of few GeVs WIMPs in the solar core (see e.g.[10] and references therein) that mimics a decrease of the opacity at the solar center.

The second term $\delta \kappa_{\mathrm{Z}}(r)$, which we refer to as composition opacity change, describes the effects of a variation of $\left\{z_{\mathrm{i}}\right\}$. It takes into account that a modification of the photospheric admixture implies a different distribution of metals inside the sun and, thus, a different opacity profile, even if the function $\kappa\left(\rho, T, Y, Z_{\mathrm{i}}\right)$ is unchanged. The contribution $\delta \kappa_{\mathrm{Z}}(r)$ is given by (see appendix $\mathrm{A}$ and $\left.\mathrm{B}\right)$ :

$$
\delta \kappa_{\mathrm{Z}}(r)=\frac{\bar{\kappa}\left(\bar{\rho}(r), \bar{T}(r), \bar{Y}(r), Z_{i}(r)\right)}{\bar{\kappa}\left(\bar{\rho}(r), \bar{T}(r), \bar{Y}(r), \bar{Z}_{i}(r)\right)}-1
$$

where $Z_{\mathrm{i}}(r)=\bar{Z}_{\mathrm{i}}(r)\left(z_{\mathrm{i}} / \bar{z}_{\mathrm{i}}\right)$ and can be calculated as:

$$
\left.\delta \kappa_{\mathrm{Z}}(r) \simeq \sum_{i} \frac{\partial \ln \bar{\kappa}}{\partial \ln Z_{i}}\right|_{\mathrm{SSM}} \delta z_{\mathrm{i}, \mathrm{b}}
$$

where $\delta z_{\mathrm{i}}$ represents the fractional variation of $z_{\mathrm{i}}$ and the symbol $\left.\right|_{\mathrm{SSM}}$ indicates that we calculate the derivatives along the density, temperature and chemical composition profiles predicted by the SSM.

Eq. (2), although being approximate, is quite useful because it makes explicit the connection (and the degeneracy) between the effects produced by a modification of the radiative opacity and of those produced by a modification of the heavy element admixture. In this paper, we take as a reference the Asplund, Grevesse, Sauval 2005 composition (AGS05) [2] and we refer with 'SSM predictions' to the numerical results obtained by using this composition as input for evolutionary solar model calculation: ${ }^{3}$.

\footnotetext{
the present sun.

${ }^{3}$ The black dashed lines in the left panel of Fig.1 has been obtained by using the FRANEC code. See [1] for a description of the code and [1] for a description of the results.
} 
Other compilation can be considered, like e.g. the Grevesse, Sauval 1998 (GS98) [5] or the more recent Asplund, Grevesse, Sauval, Scott 2009 (AGSS09) [3]. The red and blue dashed lines in the right panel of Fig.1 correspond to the opacity change $\delta \kappa_{Z}(r)$ that are obtained when we use the GS98 and the AGSS09 admixture in place of the AS05 composition, as calculated by applying rel.(5) and by using the logarithmic derivatives $\partial \ln \kappa / \partial \ln Z_{\mathrm{i}}$ presented in Fig.12 of [7]. We observe that the variation from AGS05 to GS98 (AGSS09) heavy element admixture corresponds to increasing the opacity by about $5 \%(1 \%)$ at the center of the sun and by about $20 \%(5 \%)$ at the bottom of the convective region. The effects of these opacity changes on helioseismic and solar neutrino observables, calculated in the LSM approach, are described in tab,1 and in the left panel of fig.1. They can be compared with the results of full non-linear evolutionary codes reported e.g. in [12], obtaining a satisfactory agreement.

\section{The method}

In the following, we consider the effects produced by a generic variation of the opacity profile $\delta \kappa(r)$, without discussing whether this is due to a change of the function $\kappa\left(\rho, T, Y, Z_{\mathrm{i}}\right)$ or to a change of the admixture $\left\{z_{\mathrm{i}}\right\}$. As a results of this modification, we obtain a solar model that deviates from SSM predictions. If the opacity variation is sufficiently small (i.e. $\delta \kappa(r) \ll 1$ ), the sun responds linearly. In this case, the fractional variation of a generic quantity $Q$, defined as:

$$
\delta Q=\frac{Q}{\bar{Q}}-1
$$

can be related to $\delta \kappa(r)$ by the linear relation:

$$
\delta Q=\int d r K_{Q}(r) \delta \kappa(r)
$$

The kernel $K_{Q}(r)$ represents the functional derivative with respect to opacity and allows to quantify the sensitivity of $Q$ to opacity variations in different zones of the sun.

In this paper, we determine numerically the kernels $K_{Q}(r)$ for helioseismic observables and solar neutrino fluxes, by using the linear solar model (LSM) approach presented in [1, 9] and briefly summarized in the appendix. In this approach, the structure equations of the present sun are linearized and, by estimating the (variation of) the present solar composition from the (variation of) the nuclear reaction rates and elemental diffusion efficiency in the present sun, we obtain a linear system of ordinary differential equations that can be easily solved and that completely determines the physical and chemical properties of the sun. It was shown in [1] that this kind of approach reproduces with good accuracy the results of non-linear evolutionary solar models and, thus, can be used to study in an efficient and transparent way the role of parameters and assumptions in solar model construction 4 .

The estimate of $K_{Q}(r)$ at a given point $r=r_{0}$ is obtained by performing a localized increase of opacity in the vicinity of $r_{0}$. More precisely, we calculate the variation $\delta Q\left(r_{0}\right)$ produced by a normalized 5

\footnotetext{
${ }^{4}$ The results presented in this paper can be compared with those presented in [8] where some of the kernels presented here are calculated by using static solar models and/or evolutionary models with simplified equation of state and without elemental diffusion. Where comparison is possible, a very good agreement is achieved showing that the linearization procedure adopted here and the simplifying assumptions implied in [8] do not introduce relevant errors. In order to make the comparison, one should note that the definition of the kernels given in 8 differs form that adopted in this paper.

${ }^{5}$ We remark that the LSM are linear "by construction". The validity of the the linear relation (7) is, thus, not limited by the condition $\delta \kappa(r) \ll 1$.
} 


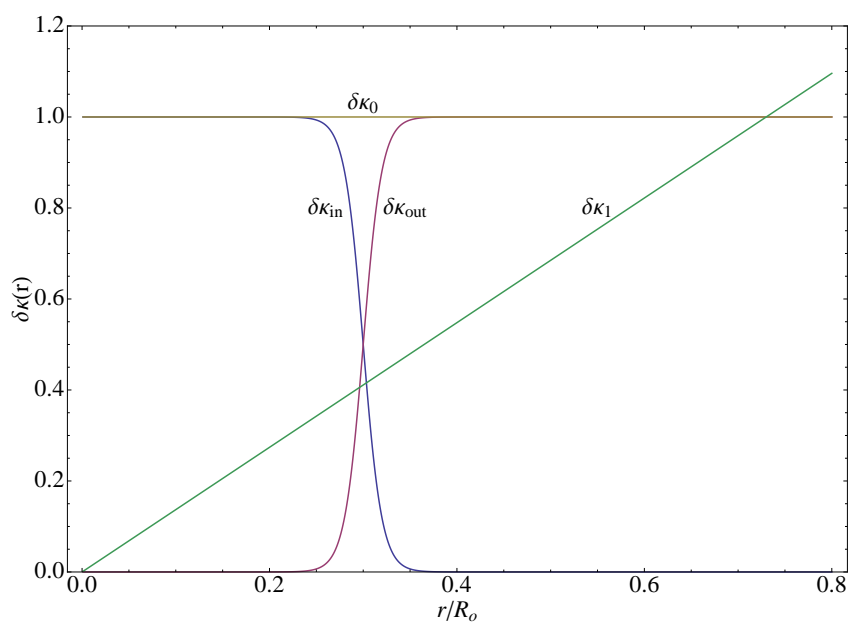

Figure 2: The functions $\delta \kappa_{\mathrm{in}}(r), \delta \kappa_{\mathrm{out}}(r), \delta \kappa_{0}(r)$ and $\delta \kappa_{1}(r)$ adopted in the parametrizations 11 12) and defined in eqs. 13.).

gaussian increase of opacity centered in $r_{0}$ :

$$
\delta \kappa(r)=G\left(r-r_{0}\right) \equiv \frac{1}{\sqrt{2 \pi} \delta r} \exp \left[-\frac{\left(r-r_{0}\right)^{2}}{2 \delta r^{2}}\right]
$$

with $\delta r=0.01 R_{\odot}$, and we assume that:

$$
K_{Q}\left(r_{0}\right)=\delta Q\left(r_{0}\right)
$$

This corresponds to the approximation:

$$
K_{Q}\left(r_{0}\right) \simeq \int d r K_{Q}(r) G\left(r-r_{0}\right)
$$

which is adequate to describe all the situations in which we consider opacity variations $\delta \kappa(r)$ which vary on scale larger than $\delta r=0.01 R_{\odot}$.

The kernels $K_{Q}(r)$ can be used to calculate the effects of an arbitrary opacity change $\delta \kappa(r)$ and allow us to discuss the role of opacity in general terms. In order to consider specific situations and to understand what kind of experimental constraints are provided by each observable quantity $Q$, it is useful, however, to consider the simple parametrizations:

$$
\begin{aligned}
& \delta \kappa(x)=A_{\text {in }} \delta \kappa_{\text {in }}(x)+A_{\text {out }} \delta \kappa_{\text {out }}(x) \\
& \delta \kappa(x)=A_{0} \delta \kappa_{0}(x)+A_{1} \delta \kappa_{1}(x)
\end{aligned}
$$

with $A_{\text {in }}, A_{\text {out }}, A_{0}$ and $A_{1}$ free adjustable parameters and:

$$
\begin{aligned}
\delta \kappa_{\text {in }}(r) & \equiv \frac{1}{\exp \left[\left(r-r_{\mathrm{c}}\right) / A\right]+1} \\
\delta \kappa_{\text {out }}(r) & \equiv 1-\frac{1}{\exp \left[\left(r-r_{\mathrm{c}}\right) / A\right]+1} \\
\delta \kappa_{0}(r) & \equiv 1 \\
\delta \kappa_{1}(r) & \equiv \frac{r}{\bar{R}_{\mathrm{b}}}
\end{aligned}
$$




\begin{tabular}{c|cccc}
$\delta Q$ & $\delta Q_{\text {in }}$ & $\delta Q_{\text {out }}$ & $\delta Q_{0}$ & $\delta Q_{1}$ \\
\hline \hline$\delta u\left(0.1 R_{\odot}\right)$ & 0.019 & -0.036 & -0.017 & -0.022 \\
$\delta u\left(0.2 R_{\odot}\right)$ & 0.052 & -0.054 & -0.0025 & -0.014 \\
$\delta u\left(0.4 R_{\odot}\right)$ & -0.084 & 0.087 & 0.0037 & 0.031 \\
$\delta u\left(0.65 R_{\odot}\right)$ & -0.16 & 0.17 & 0.011 & 0.11 \\
\hline$\Delta Y_{\mathrm{b}}$ & 0.073 & 0.069 & +0.142 & 0.062 \\
$\delta R_{\mathrm{b}}$ & 0.12 & -0.14 & -0.02 & -0.10 \\
\hline$\delta \Phi_{\mathrm{pp}}$ & -0.069 & -0.031 & -0.100 & -0.030 \\
$\delta \Phi_{\mathrm{Be}}$ & 0.85 & 0.41 & 1.26 & 0.38 \\
$\delta \Phi_{\mathrm{B}}$ & 1.93 & 0.75 & 2.68 & 0.68 \\
$\delta \Phi_{\mathrm{O}}$ & 1.65 & 0.50 & 2.15 & 0.48 \\
$\delta \Phi_{\mathrm{N}}$ & 1.14 & 0.28 & 1.43 & 0.30 \\
\hline
\end{tabular}

Table 2: The coefficients $\delta Q_{\mathrm{in}}, \delta Q_{\mathrm{out}}, \delta Q_{0}$ and $\delta Q_{1}$ defined in eq. (14), which allow to calculate the response of helioseismic and solar neutrino observables to opacity changes parameterized by eqs. (11,12).

where $r_{\mathrm{c}}=0.3 R_{\odot}, A=0.01$, while $\bar{R}_{\mathrm{b}}=0.730 R_{\odot}$ is the radius of the convective envelope predicted by SSM (see [1]). The functions $\delta \kappa_{\text {in }}(r)$ and $\delta \kappa_{\text {out }}(r)$ correspond to a constant increase of the opacity in the energy producing zone $\left(r \leq 0.3 R_{\odot}\right)$ and in the outer radiative region $\left(r \geq 0.3 R_{\odot}\right)$, see Fig.2. They have been defined in such a way that $\delta \kappa_{\text {in }}(r)+\delta \kappa_{\text {out }}(r) \equiv 1$. The function $\delta \kappa_{0}(r)$ and $\delta \kappa_{1}(r)$ correspond to a global rescaling and to a linear tilt of the opacity profile.

In linear approximation, the fractional variation $\delta Q$ produced by the opacity profiles $(1112)$ can be expressed as:

$$
\begin{aligned}
\delta Q & =A_{\text {in }} \delta Q_{\text {in }}+A_{\text {out }} \delta Q_{\text {out }} \\
\delta Q & =A_{0} \delta Q_{0}+A_{1} \delta Q_{1}
\end{aligned}
$$

where the coefficients $\delta Q_{j}$ are given by:

$$
\delta Q_{j}=\int d r K_{Q}(r) \delta \kappa_{j}(r)
$$

with $j=$ in, out, 0,1 . We report these coefficients in Tab 2 for helioseismic observables and solar neutrino fluxes.

\section{The opacity kernels}

We calculate the opacity kernels for following observable quantities: the squared isothermal sound speed $u(r) \equiv P(r) / \rho(r)$; the surface helium abundance $Y_{\mathrm{b}}$; the depth $R_{\mathrm{b}}$ of the convective envelope; the solar neutrino fluxes $\Phi_{\nu}$, where the index $\nu=\mathrm{pp}, \mathrm{Be}, \mathrm{B}, \mathrm{N}, \mathrm{O}$ refers to the neutrino producing reactions according to the usual convention. Our results are presented in the following sub-sections, together with a discussion of the present observational constraints on $\delta \kappa(r)$. 

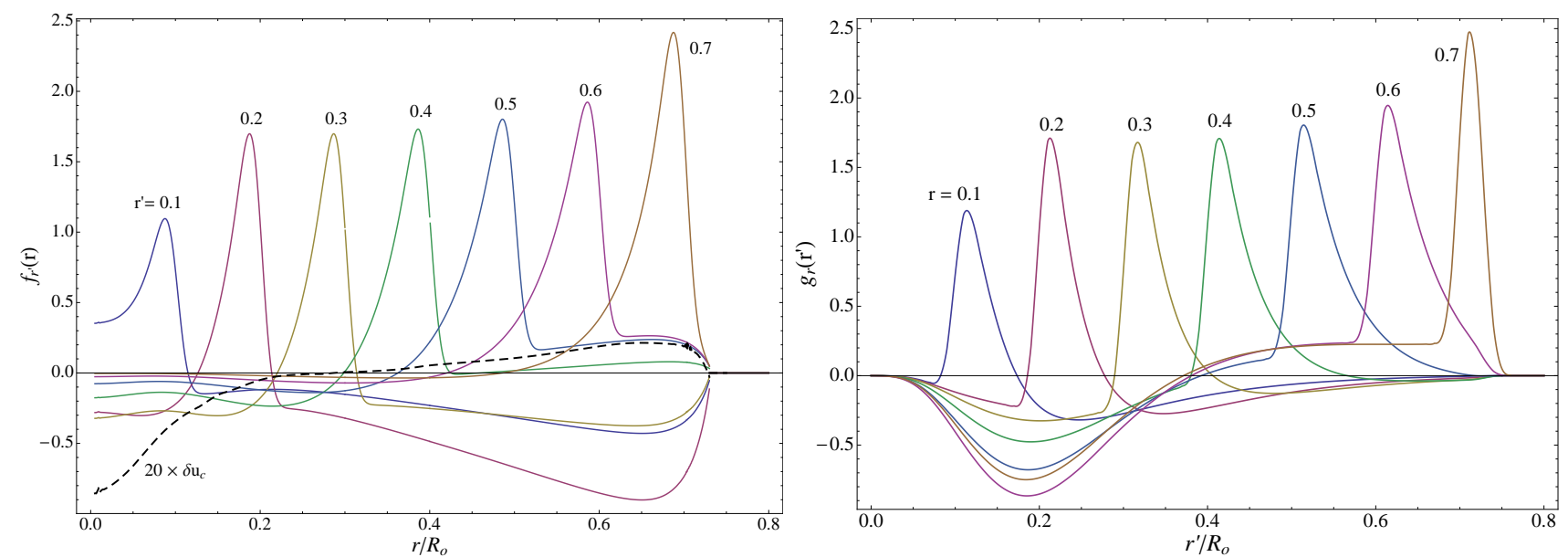

Figure 3: Left Panel: the behaviour of $f_{r^{\prime}}(r) \equiv K_{u}\left(r, r^{\prime}\right) R_{\odot}$ as a function of $r$ for the selected values of $r^{\prime}$. Right panel: the behaviour of $g_{r}\left(r^{\prime}\right) \equiv K_{u}\left(r, r^{\prime}\right) R_{\odot}$ as a function of $r^{\prime}$ for the selected values of $r$.

\subsection{The sound speed}

In Fig 3 , we discuss the properties of the sound speed kernel $K_{u}\left(r, r^{\prime}\right)$ defined by:

$$
\delta u(r)=\int d r^{\prime} K_{u}\left(r, r^{\prime}\right) \delta \kappa\left(r^{\prime}\right)
$$

where $\delta u(r)$ is the fractional variation of the squared isothermal sound speed $u(r) \equiv P(r) / \rho(r)$. In the left panel of Fig. 3, we show the functions $f_{r^{\prime}}(r) \equiv K_{u}\left(r, r^{\prime}\right) R_{\odot}$, calculated for the selected values $r^{\prime}=0.1,0.2, \ldots, 0.7 R_{\odot}$. In our approach, the functions $f_{r^{\prime}}(r)$ correspond to the sound speed variations produced (in a linear theory) by the localised opacity increases $G\left(r-r^{\prime}\right)$. One has large effects close to $r^{\prime}$ which are due to the variation of the temperature profile of the sun, as can be understood by considering that $\delta u=\delta P-\delta \rho \simeq \delta T-\delta \mu$, where $\mu$ is the mean molecular weight of the solar plasma. From eq. (57), we see that $\delta T(r)$ is expected to have a sharp decrease close to $r^{\prime}$, by an amount approximately equal to $1 / l_{t}\left(r^{\prime}\right)$, while $\delta \mu$ remains approximately constant, being related to the chemical composition of the solar plasma.

In the right panel of Fig. 3, we show the functions $g_{r}\left(r^{\prime}\right) \equiv K_{u}\left(r, r^{\prime}\right) R_{\odot}$, calculated for the selected values $r=0.1, \ldots, 0.7 R_{\odot}$, that quantify the sensitivity of the sound speed at a given $r$ to the opacity in the shell $r^{\prime}$ of the sun. They clearly indicates that $u(r)$ is maximally sensitive to the value of the opacity at $r^{\prime} \simeq r$. However, the displayed functions are different from zero everywhere, and have negative values in large part of the solar radiative region. This suggests that compensating effects can occur, especially when one considers opacity modifications that extend over a broad region of the sun.

In this respect, it is important to note that the sound speed is practically insensitive to a global rescaling of opacity. This can be appreciated by looking at the black dotted line in the left panel of Fig. 3, which is defined by:

$$
\delta u_{0}(r)=\int d r^{\prime} K_{u}\left(r, r^{\prime}\right)
$$

and corresponds to the sound speed variation produced (in a linear theory) by a constant rescaling $\delta \kappa_{0}(r) \equiv 1$. For visualization purposes, it is useful to note that the values of $\delta u_{0}(r)$ at $r=0.1, \ldots, 0.7 R_{\odot}$ correspond to the integral in $r^{\prime} / R_{\odot}$ of the functions $g_{r}\left(r^{\prime}\right)$ displayed in the right panel of Fig. 3 . We see 

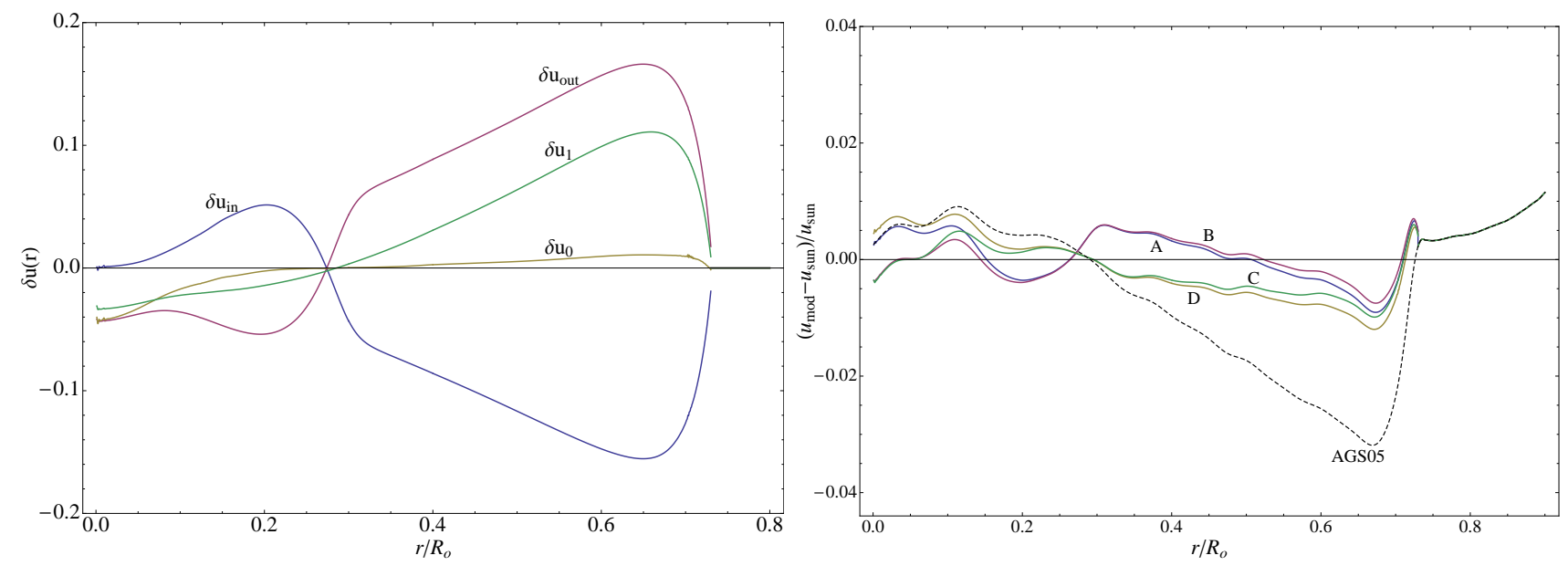

Figure 4: Left Panel: The functions $\delta u_{\mathrm{in}}(r), \delta u_{\mathrm{out}}(r), \delta u_{0}(r)$ and $\delta u_{1}(r)$ defined in eq. (18), which allow to calculate the sound speed response to opacity changes parametrized by eqs. 1112). Right panel: The difference between the sound speed inferred from helioseismic data and that obtained by solar models implementing AGS05 heavy element admixture (dotted line) with suitably modified opacity profile (the solid lines correspond to models $A, B, C, D$ described in the text).

that $\delta u_{0}(r)$ is very small, as a result of an almost perfect compensation between the positive contribution from the region $r^{\prime} \simeq r$ and the negative contribution from the other regions of the sun.

A qualitative argument to explain the stability of the sound speed is the following. The virial theorem connects the gravitational energy, $E_{g}=-\int d m G m / r$, and the thermal energy content of a given star, $E_{i}=3 / 2 \int d m(P / \rho)=3 / 2 \int d m u$, being $E_{g}=-2 E_{i}$. For a generic star, a global rescaling of the opacity reflects into a global rescaling of the radial profile and, thus, taking into account the virial theorem, also into a global rescaling of the sound speed. This is not the case for the sun because the solar radius is observationally determined. We are forced to re-adjust the free parameters in the model in order to keep the solar radius fixed, with the effect of stabilizing the radial profile and, thus, the sound speed profile $u(r)$.

The above result has relevant implications. In particular, the statement that the "sound speed problem" requires an increase of the opacity at the bottom of the convective region is, strictly speaking, not correct because other solutions are possible. We can consider, e.g, the sound speed profiles produced by the opacity variations parametrized by eqs. (11 12). In linear approximation, we have that:

$$
\begin{aligned}
& \delta u(r)=A_{\text {in }} \delta u_{\text {in }}(r)+A_{\text {out }} \delta u_{\text {out }}(r) \\
& \delta u(r)=A_{0} \delta u_{0}(r)+A_{1} \delta u_{1}(r) .
\end{aligned}
$$

The functions $\delta u_{\text {in }}(r), \delta u_{\text {out }}(r), \delta u_{0}(r)$ and $\delta u_{1}(r)$ are shown in the left panel of Fig. 4 and are reported in Tab. 2 for the selected values $r=0.1,0.2,0.4,0.65 R_{\odot}$. We see that $\delta u_{0}(r) \ll \delta u_{1}(r)$, indicating that the "tilt" and not the scale of the opacity is fixed by the sound speed. Moreover, we have $\delta u_{\text {in }}(r) \simeq-\delta u_{\text {out }}(r)$ that shows that the effect of an enhancement of the opacity in the external radiative region can be equally produced by a decrease of the opacity at the solar center.

This is confirmed in the right panel of Fig 4 where we show the sound speed profiles obtained by implementing four different opacity modifications, described by:

Model A: $15 \%$ decrease of opacity in the energy producing region $\left(A_{\text {in }}=-0.15\right.$ and $\left.A_{\text {out }}=0\right)$; 
Model B: $15 \%$ increase of the opacity in the outer radiative region $\left(A_{\mathrm{in}}=0\right.$ and $\left.A_{\text {out }}=0.15\right)$;

Model $C$ : a linear tilt of opacity corresponding to a $20 \%$ increase at the bottom of the convective envelope $\left(A_{0}=0\right.$ and $\left.A_{1}=0.2\right)$;

Model D: a linear tilt plus a global rescaling of opacity corresponding to a $20 \%$ decrease at the solar center $\left(A_{0}=-0.2\right.$ and $\left.A_{1}=0.2\right)$.

The sound speed profiles obtained in all the considered cases reproduce equally well the result inferred by helioseismic data, showing that the helioseismic determination of $u(r)$ translates into a bound on the differential increase $A_{\text {out }}-A_{\text {in }} \simeq 0.15$ and on the tilt $A_{1} \simeq 0.2$, with no relevant constraint on $A_{0}$ (or, equivalently, $A_{\text {in }}+A_{\text {out }}$ ) that fix the global scale of opacity.

In light of this observation, it is intriguing the possibility that non-standard effects that mimic a decrease of the opacity at the solar center, like e.g. the accumulation of few GeVs WIMPs in the solar core (see e.g. [10]), could have a role in the solution of the solar composition puzzle. We will see, in the following, that this possibility is disfavoured by the determination of the surface helium abundance and by the measurement of the ${ }^{7} \mathrm{Be}$ and ${ }^{8} \mathrm{~B}$ neutrino fluxes ${ }^{6}$

\subsection{The surface helium abundance}

In the left panel of Fig 5 , we show with solid line the functional derivative $K_{Y}(r)$ of the surface helium abundance $Y_{\mathrm{b}}$, defined according to equation:

$$
\Delta Y_{\mathrm{b}}=\int d r K_{\mathrm{Y}}(r) \delta \kappa(r)
$$

where we considered the absolute variation $\Delta Y_{\mathrm{b}}$ to conform with the notations adopted in [1]

The surface helium abundance depends on the initial chemical composition and on the effects of elemental diffusion. In ref.[1], by assuming that (the variation of) the time-integrated effect of diffusion can be estimated from (the variation of) diffusion efficiency in the present sun, we have obtained the following relation:

$$
\Delta Y_{\mathrm{b}}=A_{Y} \Delta Y_{\mathrm{ini}}+A_{C} \delta C
$$

with $A_{Y}=0.838$ and $A_{C}=0.033$, which gives $\Delta Y_{\mathrm{b}}$ as a function of the absolute variation of the initial helium abundance, $\Delta Y_{\mathrm{ini}}$, and of the fractional variation of pressure at the bottom of the convective region, $\delta C=\delta P_{\mathrm{b}}$. The above equation allows us to obtain the functional derivative $K_{Y}(r)$ as the sum of two contributions, one related to the term $A_{Y} \Delta Y_{\text {ini }}$ and the other to $A_{\mathrm{C}} \delta C$. These are shown in Fig 5 with red and blue dashed lines, respectively.

The function $K_{\mathrm{Y}}(r)$ is positive everywhere, showing that an increase of opacity in an arbitrary shell of the sun translates into an increase of the helium abundance 8 . Moreover, the kernel $K_{Y}(r)$ has a rather broad profile. This implies that the determination of $Y_{\mathrm{b}}$ effectively constrains the opacity scale and breaks the degeneracy between the possible solutions of the sound speed problem presented

\footnotetext{
${ }^{6}$ The idea that the accumulation of few GeV WIMPs in the solar core could alleviate the "solar composition problem" was originally proposed by [13. The recent paper [14] presented a qualitative implementation of this idea. The effect on boron neutrinos is discussed in [15].

${ }^{7}$ Here and in the following, we use the notation $Q_{\mathrm{b}}$ to indicate that a given quantity $Q(r)$ is evaluated at the bottom of the convective region, i.e. $Q_{\mathrm{b}} \equiv Q\left(\bar{R}_{\mathrm{b}}\right)$ where $\bar{R}_{\mathrm{b}}=0.730 R_{\odot}$.

8 If the chemical composition is fixed, an increase of the opacity implies a decrease of the total luminosity $L$ which roughly scales as $L \propto \mu^{4} / \kappa$, where $\mu$ is the mean molecular weight. In order to reproduce the observed solar luminosity $L_{\odot}$, we are forced to readjust the chemical composition of the sun by increasing the helium abundance. This has the simultaneous effects of increasing $\mu$ and decreasing $\kappa$.
} 

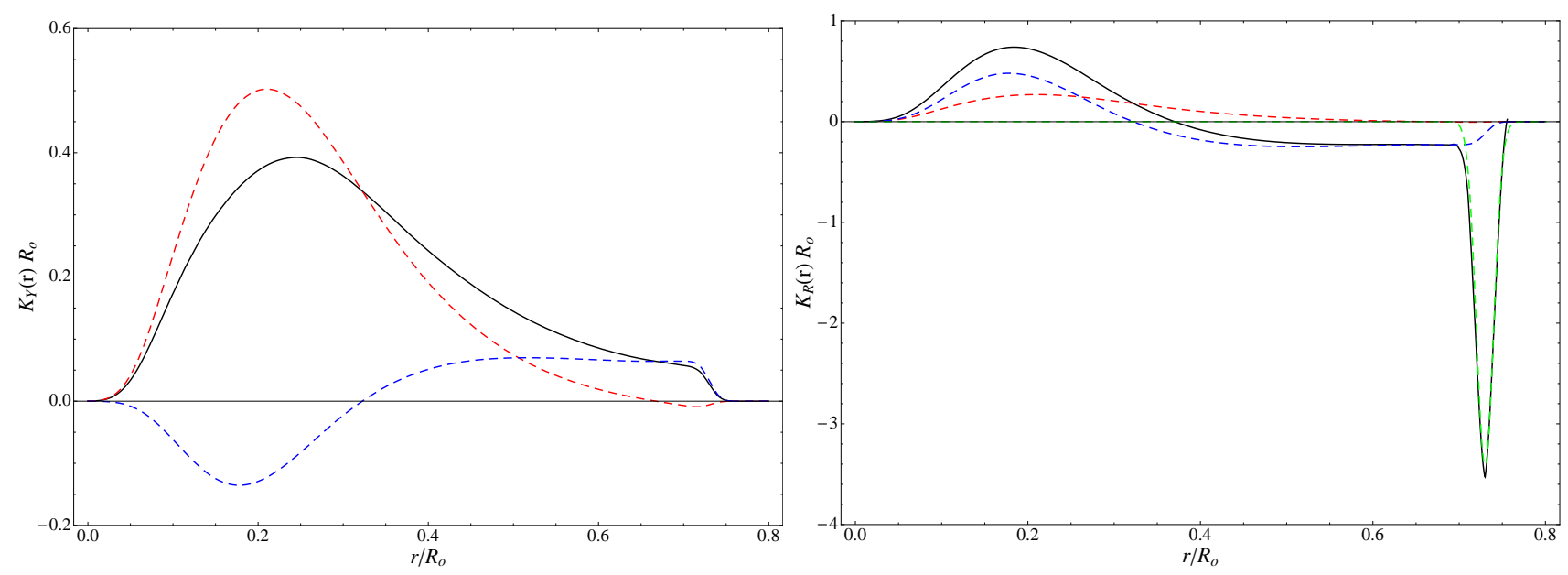

Figure 5: Left Panel: The solid line corresponds to the kernel $K_{Y}(r)$ defined in eq. (19). The red and blue dashed lines describe the contributions to surface helium variations provided by the terms $A_{Y} \Delta Y_{\mathrm{ini}}$ and $A_{C} \delta C$, respectively. See text for details. Right Panel: The solid line corresponds to the kernel $K_{R}(r)$ defined in eq. 23). The red, blue and green dashed lines describe the contributions to convective radius variations provided by the terms $\Gamma_{Y} \Delta Y_{\mathrm{ini}}, \Gamma_{C} \delta C$ and $\Gamma_{\kappa} \delta \kappa_{\mathrm{b}}$, respectively. See text for details.

in the previous section. The SSM that implements the AGS05 heavy element admixture predicts the value $\bar{Y}_{\mathrm{b}}=0.229$ (see e.g. [1]) which is about $6 \sigma$ lower than the helioseismic determination $Y_{\mathrm{b}}=0.2485 \pm 0.0034$ [7]. This discrepancy requires an increase of the opacity, as it can be obtained, e.g., by increasing the metal content of the sun. Models that accounts for a reduction "effective" opacity at the solar center are expected to decrease $Y_{\mathrm{b}}$, increasing the disagreement with helioseismic results.

A simple quantitative analysis can be performed by considering the opacity profiles 1112 that produce the variations $\Delta Y_{\mathrm{b}}$ given by:

$$
\begin{aligned}
& \Delta Y_{\mathrm{b}}=0.073 A_{\text {in }}+0.069 A_{\text {out }} \simeq 0.07\left(A_{\text {in }}+A_{\text {out }}\right) \\
& \Delta Y_{\mathrm{b}}=0.142 A_{0}+0.062 A_{1}
\end{aligned}
$$

By comparing the SSM prediction with the helioseismic result, we obtain $A_{\text {in }}+A_{\text {out }}=0.28 \pm 0.05$ that, combined with the "orthogonal" constraint $A_{\text {out }}-A_{\text {in }} \simeq 0.15$ provided by the sound speed, gives $A_{\text {in }}=0.04 \div 0.09$ and $A_{\text {out }}=0.19 \div 0.24$. Alternatively, we can use eq. 22 and the information on the tilt $A_{1} \simeq 0.2$ provided by the sound speed, to fix the opacity at the solar center obtaining $A_{0}=0.035 \div 0.075$.

\subsection{The convective radius}

In the right panel of Fig 5 , we show with a solid line the functional derivative $K_{R}(r)$ of the convective radius $R_{\mathrm{b}}$ defined according to the relation:

$$
\delta R_{\mathrm{b}}=\int d r K_{\mathrm{R}}(r) \delta \kappa(r)
$$

We see that the kernel $K_{R}(r)$ has a very sharp peak at $r \simeq \bar{R}_{\mathrm{b}}=0.730 R_{\odot}$ that reflects the (well-known) fact that the convective radius is particularly sensitive to the opacity at the bottom of the convective 
region. The shape of this peak has not a precise physical meaning and depends on the method of calculation. The effect of opacity changes has been, in fact, estimated within the LSM approach, in which the fractional variation $\delta R_{\mathrm{b}}$ is calculated from:

$$
\delta R_{\mathrm{b}}=\Gamma_{Y} \Delta Y_{\mathrm{ini}}+\Gamma_{C} \delta C+\Gamma_{\kappa} \delta \kappa_{\mathrm{b}}
$$

where $\Gamma_{Y}=0.449, \Gamma_{C}=-0.117$ and $\Gamma_{\kappa}=-0.085$, while $\delta \kappa_{\mathrm{b}}$ is fractional variation of opacity at the bottom of the convective envelope, i.e. $\delta \kappa_{\mathrm{b}}=\delta \kappa\left(\bar{R}_{\mathrm{b}}\right)$. The "local" term $\Gamma_{\kappa} \delta \kappa_{\mathrm{b}}$ translates into a delta-function contribution $\Gamma_{\kappa} \delta\left(r-\bar{R}_{\mathrm{b}}\right)$ to the functional derivative. Since we evaluate numerically the kernel by applying a localised gaussian increase of opacity, this is convolved with the function $G\left(r-r_{0}\right)$ given in eq. (8). As a final result, one obtains the contribution $\Gamma_{\kappa} G\left(r-\bar{R}_{\mathrm{b}}\right)$ to the kernel $K_{R}(r)$ which is shown by the green dashed line in Fig.5, whereas the red and blue dashed lines describe the contributions arising from $\Gamma_{Y} \Delta Y_{\text {ini }}$ and $\Gamma_{C} \delta C$, respectively. We remark that the area under the peak at $r=\bar{R}_{\mathrm{b}}$ is approximately equal to $\Gamma_{\kappa}$ and does not depend on the calculation method. We can, thus, safely use the functional derivative $K_{R}(r)$ to describe all the situations in which opacity varies on scale larger than $\delta r=0.01 R_{\odot}$.

Eqs. 20 and (24) can be combined to obtain a direct determination of $\delta \kappa_{\mathrm{b}}$ from quantities that are all determined by helioseismic observations. We can, in fact, eliminate $\Delta Y_{\text {ini }}$ from eq.24, obtaining:

$$
\delta \kappa_{\mathrm{b}}=C_{Y} \Delta Y_{\mathrm{b}}+C_{\mathrm{R}} \delta R_{\mathrm{b}}+C_{\rho} \delta \rho_{\mathrm{b}}
$$

where:

$$
\begin{aligned}
C_{Y} & =-\frac{\Gamma_{Y}}{A_{Y} \Gamma_{\kappa}}=6.27 \\
C_{\mathrm{R}} & =\frac{1}{\Gamma_{\kappa}}=-11.71 \\
C_{\rho} & =\frac{1}{\Gamma_{\kappa}}\left[\frac{A_{C} \Gamma_{Y}}{A_{Y}}-\Gamma_{C}\right]=-1.58
\end{aligned}
$$

In the derivation of the above result, we have considered that $\delta C=\delta P_{\mathrm{b}} \simeq \delta \rho_{\mathrm{b}}$, since the fractional variation of the sound speed $\delta u(r)$ is expected to vanish at the bottom of the convective region, i.e. $\delta u_{\mathrm{b}}=\delta P_{\mathrm{b}}-\delta \rho_{\mathrm{b}} \simeq 0$, as it is discussed in [1]. The discrepancy between the helioseismic determinations of $R_{\mathrm{b}}$ and $Y_{\mathrm{b}}$ and the predictions of SSMs implementing AS05 admixture is quantified as $\delta R_{\mathrm{b}}=$ $-0.0205 \pm 0.0015$ and $\Delta Y_{\mathrm{b}}=0.0195 \pm 0.0034$. The density at the bottom of the convective region deviates from value inferred by helioseismology by $\delta \rho_{\mathrm{b}}=0.08$, as it is discussed e.g. in [12]. We obtain $\delta \kappa_{\mathrm{b}} \simeq 0.24 \pm 0.03$, where errors have been combined in quadrature and we have neglected the (subdominant) contribution to the total error budget due to the uncertainties in the density determination. We remark that the obtained results is model-independent, since it does not rely on any assumption or parametrization for the function $\delta \kappa(r)$.

As a final application, we consider the response of $R_{\mathrm{b}}$ to the opacity changes parametrized by eqs. 11 12). We obtain the relations

$$
\begin{aligned}
& \delta R_{\mathrm{b}}=0.12 A_{\text {in }}-0.14 A_{\text {out }} \simeq 0.13\left(A_{\text {in }}-A_{\text {out }}\right) \\
& \delta R_{\mathrm{b}}=-0.02 A_{0}-0.10 A_{1}
\end{aligned}
$$

which show that the convective radius, just like the sound speed, provides bounds on the differential increase $A_{\text {out }}-A_{\text {in }}$ and on tilt $A_{1}$, with no relevant constraints on $A_{0}$ (or equivalently $A_{\text {in }}+A_{\text {out }}$ ) that 

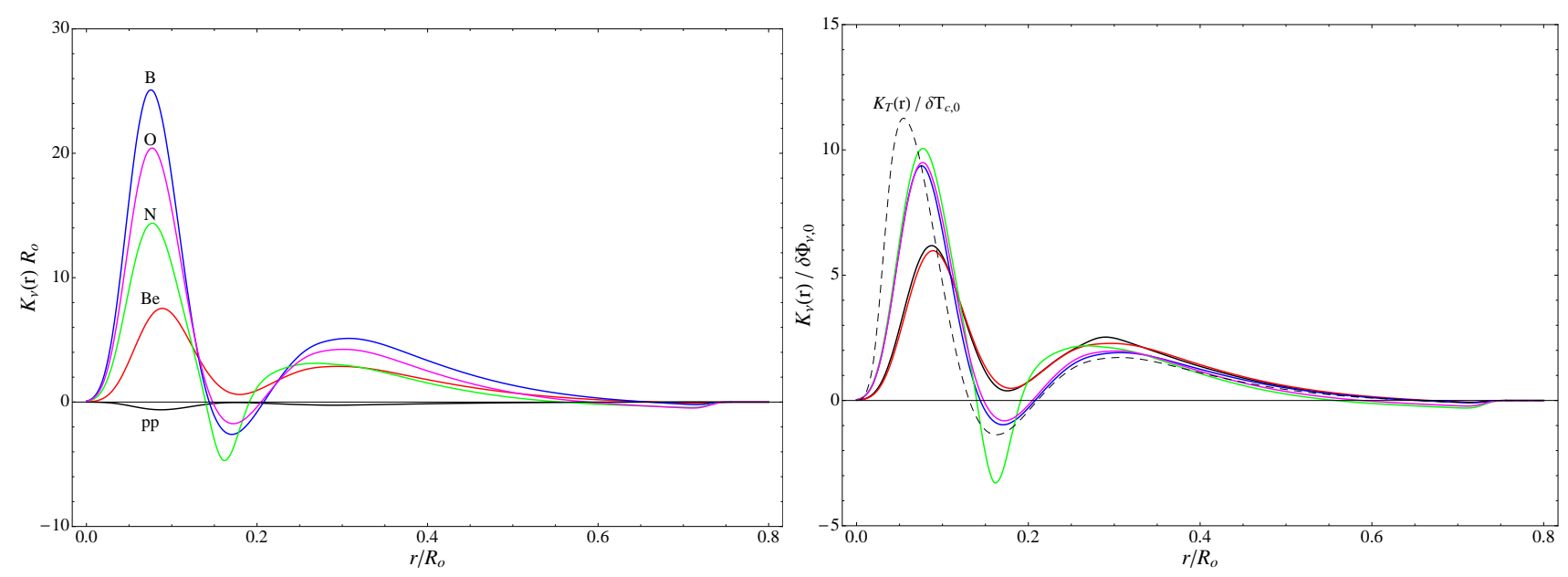

Figure 6: Left Panel: The solar neutrino kernels $K_{\nu}(r)$ defined in eq. (31). Right Panel: The solid lines are the normalized solar neutrino kernels $K_{\nu}(r) / \delta \Phi_{\nu, 0}$. The dashed line shows the normalized kernel $K_{T}(r) / \delta T_{\mathrm{c}, 0}$ defined in eq.(35), that describes the response of the solar central temperature to localised opacity modifications.

fix the global scale of opacity. By considering $\delta R_{\mathrm{b}}=-0.0205 \pm 0.0015$, we obtain $A_{\text {out }}-A_{\text {in }} \sim 0.15$ and $A_{1} \sim 0.2$ in substantial agreement (and complete degeneracy) with the information provided by the sound speed measurement. By combining this information with the constraints provided by the surface helium abundance and performing a simple $\chi^{2}$ analysis, we obtain $A_{\text {in }}=0.07 \pm 0.04$ and $A_{\text {out }}=0.21 \pm 0.04$ for the parametrization given in eq. (11) and $A_{0}=0.056 \pm 0.040$ and $A_{1}=0.187 \pm 0.023$ for that given in eq. (12). The corresponding bounds on the opacity change $\delta \kappa(r)$ are shown in fig. (8) and commented in the conclusive section.

\subsection{Neutrino Fluxes}

In the left panel of Fig 6 , we show the functional derivatives $K_{\nu}(r)$ of the neutrino fluxes $\Phi_{\nu}$ defined according to relation:

$$
\delta \Phi_{\nu}=\int d r K_{\nu}(r) \delta \kappa(r)
$$

where the index $\nu=\mathrm{pp}, \mathrm{Be}, \mathrm{B}, \mathrm{N}$, O labels the neutrino producing reactions according to the usual convention. The kernel $K_{\nu}(r)$ have been calculated by using the LSM approach and by taking into account that the fractional variations of the fluxes $\delta \Phi_{\nu}$ are related to physical and chemical properties of the sun by:

$$
\delta \Phi_{\nu}=\int d r\left[\phi_{\nu, \rho}(r) \delta \rho(r)+\phi_{\nu, T}(r) \delta T(r)+\phi_{\nu, Y}(r) \Delta Y(r)+\phi_{\nu, Z}(r) \delta Z(r)\right]
$$

The functions $\phi_{\nu, j}(r)$ have been defined and calculated in the fig. 10 of [1].

Our results show that neutrino fluxes probe the opacity of the sun in the region $r \leq 0.45 R_{\odot}$. The kernels $K_{\mathrm{B}}(x), K_{\mathrm{Be}}(x), K_{\mathrm{N}}(x)$ and $K_{\mathrm{O}}(x)$ are positive-valued almost everywhere, while the kernel $K_{\mathrm{pp}}(r)$ is negative. This indicates that an increase of opacity generally translates into an enhancement of ${ }^{8} \mathrm{~B},{ }^{7} \mathrm{Be}$ and $\mathrm{CNO}$ neutrino fluxes and into a (slight) decrease of the pp-neutrino component. In Tab. 2, we show the coefficients $\delta \Phi_{\nu, \text { in }}, \delta \Phi_{\nu, \text { out }}, \delta \Phi_{\nu, 0}$ and $\delta \Phi_{\nu, 1}$ that allows to describe the effects of 
opacity changes parametrized by eqs. (11) 12 , through the simple relations:

$$
\begin{aligned}
& \delta \Phi_{\nu}=A_{\text {in }} \delta \Phi_{\nu, \text { in }}+A_{\text {out }} \delta \Phi_{\nu, \text { out }} \\
& \delta \Phi_{\nu}=A_{0} \delta \Phi_{\nu, 0}+A_{1} \delta \Phi_{\nu, 1} .
\end{aligned}
$$

We see that ${ }^{8} \mathrm{~B}$ and $\mathrm{CNO}$ neutrinos are extremely sensitive to opacity changes, as it is expected since they strongly depend on the temperature of the central regions of the sun. It is interesting to note in the right panel of Fig. 6 that the normalized neutrino kernels $K_{\nu}(x) / \Phi_{\nu, 0}$ have a common behaviour, with two maxima at $r \sim 0.1 R_{\odot}$ and $r \sim 0.3 R_{\odot}$, and one minimum at $r \sim 0.2 R_{\odot}$. This shows that different fluxes basically probe the same quantity, constraining the opacity profile with the maximal sensitivity in the two regions of the sun $r \sim 0.05 \div 0.15$ and $r \sim 0.2 \div 0.45$.

Some insights on the above results can be obtained by recalling that the total neutrino flux is essentially fixed by the solar luminosity constraint. We, thus, expect that $\Delta \Phi_{\text {tot }}=\sum_{\nu} \bar{\Phi}_{\nu} \cdot \delta \Phi_{\nu} \simeq$ 0 , where $\bar{\Phi}_{\nu}$ are the SSM predictions for the various neutrino components. Considering that about $99 \%$ of the total flux is provided by pp and Be neutrinos, the luminosity constraint implies $\delta \Phi_{\mathrm{pp}} \simeq$ $-\left(\bar{\Phi}_{\mathrm{Be}} / \bar{\Phi}_{\mathrm{pp}}\right) \delta \Phi_{\mathrm{Be}}=-0.075 \delta \Phi_{\mathrm{Be}}$ which explains the smallness of the pp-neutrino kernel, the ratio between the pp and Be-neutrino coefficients in tab. 2 and the equality between the normalized pp and Be-neutrino kernels observed in the right panel of Fig. 6. The wavy shape of the kernels $K_{\nu}(r)$ reflects, instead, the response of central temperature to localised opacity modifications. This was first noted and discussed by [8] and it is seen in the right panel of Fig. 6, where we show with a dashed line the functional derivative of the central temperature $T_{\mathrm{c}}$, defined by:

$$
\delta T_{\mathrm{c}}=\int d r K_{T}(r) \delta \kappa(r)
$$

For convenience, we plot the normalized kernel $K_{\mathrm{T}}(x) / \delta T_{\mathrm{c}, 0}$, where the normalization factor is $\delta T_{\mathrm{c}, 0}=$ 0.138

The peculiar behaviour with $r$ of the kernel $K_{T}(r)$ cannot be explained in simple terms. A qualitative comprehension can be obtained from Fig. 7, where we show the temperature profiles $\delta T(r)$ produced by the opacity changes $\delta \kappa(r)=G\left(r-r_{0}\right)$ with $r_{0}=0.1,0.2,0.3 R_{\odot}$. We see that the performed opacity modifications translate into a large increase of temperature close to $r_{0}$, that necessarily alters nuclear burning rates since $r_{0}$ is inside or close to the energy producing region. The free parameters of the solar model are readjusted in such a way that the same luminosity is obtained with a different temperature profile.

In the two cases $r_{0}=0.1 R_{\odot}$ (i.e. opacity increase well inside the energy producing region) and $r_{0}=0.3 R_{\odot}$ (i.e. opacity increase just outside the energy producing region), the entire energy producing zone is affected. A new "equilibrium" situation is achieved in which nuclear burnings occur at higher temperatures with a larger helium abundance, favoring ${ }^{8} \mathrm{~B}$, CNO and ${ }^{7} \mathrm{Be}$ neutrinos at expenses of pp-neutrinos. For $r_{0}=0.2 R_{\odot}$, we have a peculiar situation since the maximal effect is produced in a region of the sun where only pp neutrinos are produced, as it is seen from fig.10 of [1]. This necessarily shifts energy production outwards with respect to the SSM case. In order to avoid overproduction of energy and to recover the observed luminosity, the central temperature $T_{\mathrm{c}}$ has to be (slightly) decreased so that the burning rates at the center of the sun are suppressed.

At present, the best studied components of the solar neutrino flux are the ${ }^{8} \mathrm{~B}$ neutrino flux which is determined by the SNO neutral current measurement with about $6 \%$ accuracy, $\Phi_{\mathrm{B}}=(5.18 \pm 0.29) \times$ $10^{6} \mathrm{~cm}^{-2} \mathrm{~s}^{-1}$ [16], and the ${ }^{7}$ Be neutrino flux which is measured by Borexino, $\Phi_{\mathrm{Be}}=(5.18 \pm 0.51) \times$ $10^{9} \mathrm{~cm}^{-2} \mathrm{~s}^{-1}$ [17]. These fluxes have to be compared with the results of theoretical calculations. SSMs 


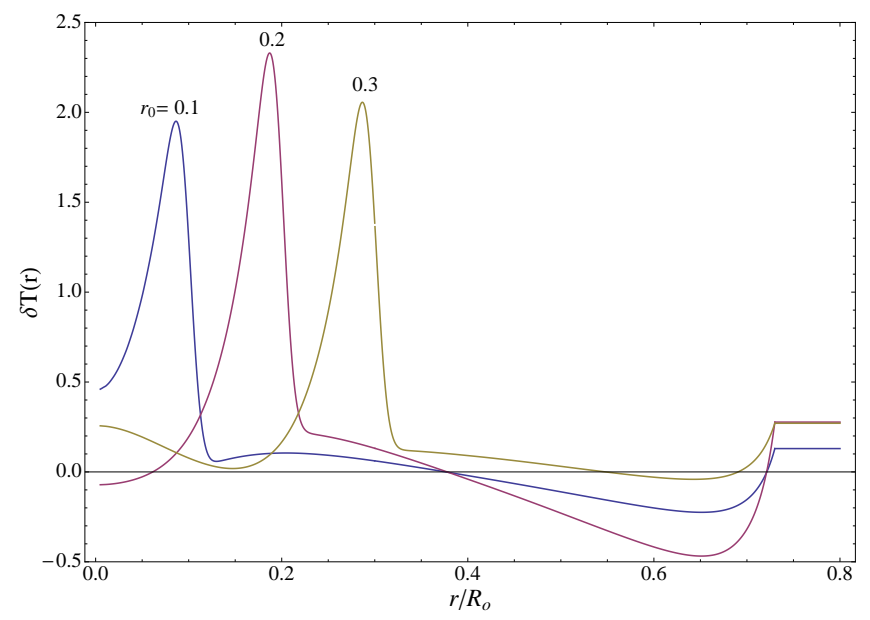

Figure 7: The temperature profile variations $\delta T(r)$ produced (in LSM) by the localized gaussian increases of opacity $G\left(r-r_{0}\right)$ defined in eq.(8) with $r_{0}=0.1,0.2,0.3 R_{\odot}$.

implementing AGS05 heavy elements admixture predict values for $\Phi_{\mathrm{B}}$ and $\Phi_{\mathrm{Be}}$ which are about $10 \%$ lower than the experimental results. We take, for definiteness, the values $\bar{\Phi}_{\mathrm{B}}=4.66 \times 10^{6} \mathrm{~cm}^{-2} \mathrm{~s}^{-1}$ and $\bar{\Phi}_{\mathrm{Be}}=4.54 \times 10^{9} \mathrm{~cm}^{-2} \mathrm{~s}^{-1}$ obtained in [12] which are affected by $\sim 9 \%$ and $\sim 5 \%$ theoretical uncertainties 9 , respectively. The difference between the theoretical predictions and the experimental data points toward a moderate increase of the central opacity of the sun. As an example, a $5 \%$ increase of the opacity in the region $r \leq 0.3 R_{\odot}$ would produce a $9.7 \%(4.3 \%)$ increase of the ${ }^{8} \mathrm{~B}\left({ }^{7} \mathrm{Be}\right)$ neutrino flux. Models that account for a reduction of the "effective" central opacity, due e.g. to WIMP accumulation, increase the disagreement and are, thus, disfavoured by solar neutrino data.

We, finally, discuss the constraints provided by solar neutrinos on opacity changes parametrized by Eqs. (11 12 . We note that the coefficients $A_{\text {in }}$ and $A_{\text {out }}$ and/or $A_{0}$ and $A_{1}$ required to fit helioseismic results (see the previous section), produce enhancements of the ${ }^{8} \mathrm{~B}$ and ${ }^{7} \mathrm{Be}$ neutrino fluxes which are equal to $28 \%$ and $14 \%$, respectively. While the ${ }^{7} \mathrm{Be}$ component would be consistent with the observational data, the ${ }^{8} \mathrm{~B}$ neutrino flux is too large with respect to the SNO measurement. When we fit simultaneously helioseismic and solar neutrino data, we obtain a slight reduction of the required opacity change. A simple $\chi^{2}$-analysis gives $A_{\text {in }}=0.05 \pm 0.03, A_{\text {out }}=0.19 \pm 0.03$ for the parameterization (11) and $A_{0}=0.038 \pm 0.034, A_{1}=0.192 \pm 0.023$ for the parameterization (12) with the best fit values corresponding to $\chi_{\min }^{2} /$ d.o.f. $=2.1 / 2$ and $\chi_{\min }^{2} /$ d.o.f. $=1.7 / 2$, respectively. The corresponding bounds on $\delta \kappa(r)$ are displayed in fig. 8.

\section{Summary}

In this paper, motivated by the solar composition problem and by using the recently developed LSM approach [1, we have discussed the effects of arbitrary opacity changes on the sun. Our main results can be summarized as it follow:

i) We have discussed the relation between the effects produced by a variation of the heavy element admixture and those produced by a modification of the radiative opacity. We have shown that the

\footnotetext{
${ }^{9}$ The quoted theoretical uncertainties are obtained from [18. In our estimate, we have not included the contribution due to opacity, since this is considered as a free parameter in our analysis.
} 

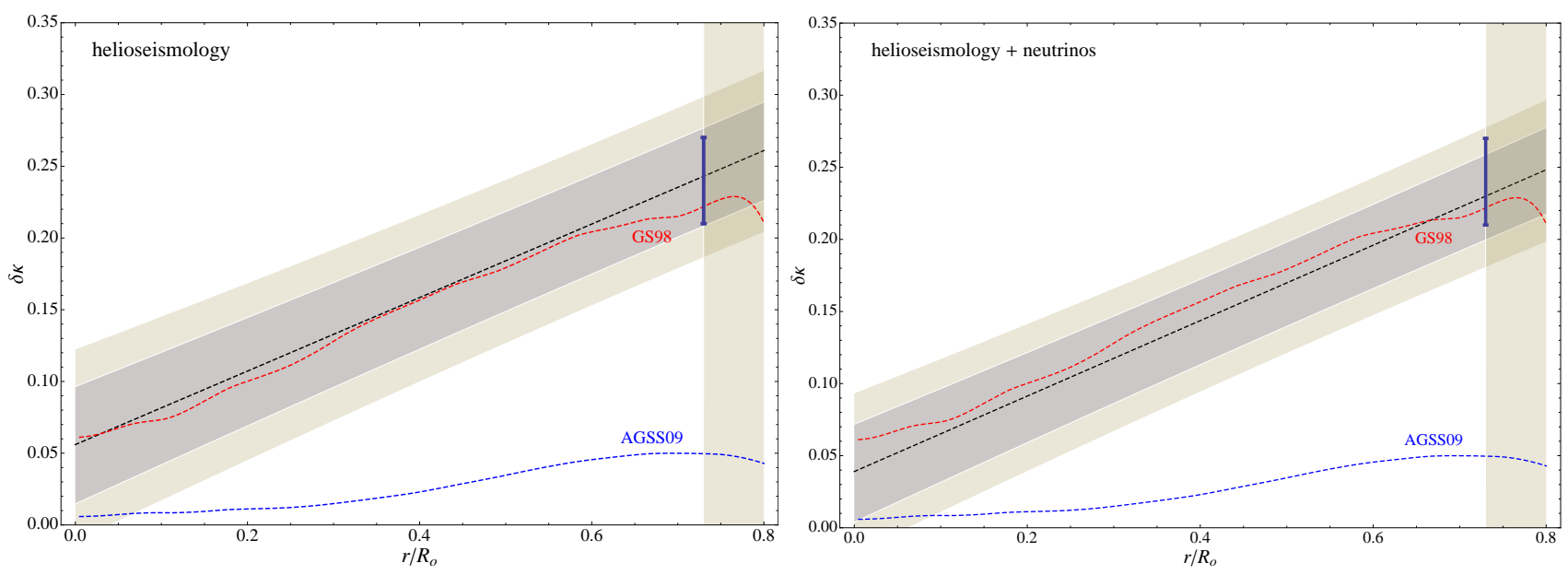

Figure 8: Left Panel: The constraints on the opacity profile $\delta \kappa(r)$ obtained from helioseismic data. Right panel: The constraints on the opacity profile $\delta \kappa(r)$ obtained from helioseismic and solar neutrino data. See text for details.

relevant quantity is the variation of the opacity profile $\delta \kappa(r)$ defined in eq. $(2)$, that is approximately given by the superposition of the intrinsic opacity change $\delta \kappa_{\mathrm{I}}(r)$ and the composition opacity change $\delta \kappa_{\mathrm{Z}}(r)$, defined in eqs.(3) and (5) respectively.

ii) We have studied the response of the sun to an arbitrary modification of the opacity $\delta \kappa(r)$. Namely, we have calculated numerically the kernels that, in a linear approximation, relate the opacity change $\delta \kappa(r)$ to the corresponding modifications of the solar observable properties. We have considered the following observable quantities: the squared isothermal sound speed $u(r)$; the surface helium abundance $Y_{\mathrm{b}}$; the convective radius $R_{\mathrm{b}}$; the solar neutrino fluxes $\Phi_{\nu}$.

iii) We have shown that different observable quantities probe different regions of the sun. Moreover, effects produced by variations of opacity in distinct zones of the sun may compensate among each other. In this respect, we noted that the sound speed $u(r)$ and the depth of the convective envelope $R_{\mathrm{b}}$ are practically insensitive to a global rescaling of the opacity.

iv) As a consequence of the above result, we have seen that the discrepancy between the SSM predictions for $u(r)$ and $R_{\mathrm{b}}$ and the helioseismic inferred values can be equally solved by a $\sim 15 \%$ decrease of the opacity a the center of the sun or by a $\sim 15 \%$ increase of the opacity in the external radiative region. The degeneracy between these two possible solutions is broken by the "orthogonal" information provided by the measurements of the surface helium abundance and of the boron and beryllium neutrino fluxes, that fix the scale of opacity and indicate that only the second possibility can effectively solve the solar composition problem.

v) We have derived a model-independent relation, eq. (25), that allows to obtain a direct determination of the opacity at the bottom of the convective envelope $\delta \kappa_{\mathrm{b}}$ from quantities that are determined by helioseimic observations, i.e. the surface helium abundance $\Delta Y_{\mathrm{b}}$, the convective radius $\delta R_{\mathrm{b}}$ and the density at the bottom of the convective region $\delta \rho_{\mathrm{b}}$. By considering the present observation values, we have obtained $\delta \kappa_{\mathrm{b}}=0.24 \pm 0.03$.

A conclusive view of the present constraints on $\delta \kappa(r)$ is contained in Fig. 8. The red (blue) dashed lines correspond to the composition opacity changes obtained when we replace the AGS05 composition with the GS98 (AGSS09) admixture. The blue tick at $r=\bar{R}_{\mathrm{b}}$ is the value of the opacity at the bottom of the convective region that is obtained by applying the model-independent rel.(25) to helioseismic data. 
The dark and light areas individuate the opacity changes $\delta \kappa(r)$ that, for each value of $r$, are obtained at $68.3 \%(1 \sigma)$ and $95.4 \%(2 \sigma)$ C.L. by applying a simple $\chi^{2}$ analysis to the opacity modification parametrized by eq. (12). The left panel takes into account only helioseismic observables. Namely, it is obtained by using the present observational determinations of the surface helium abundance and of the convective radius. The selected opacity profile $\delta \kappa(r)$ is also consistent with the helioseismic determination of the sound speed, as it is has been discussed in fig. 4 . The right panel includes also the observational information on boron and beryllium neutrinos. These data move the required opacity change $\delta \kappa(r)$ towards slightly smaller values. In summary, we see that the helioseismic and solar neutrino data select an opacity profile that well corresponds to the GS98 heavy element admixture. They clearly disfavour different solutions of the solar composition problem, like e.g. that recently proposed by [14].

\section{Acknowledgments}

It is a pleasure for me to thank B. Ricci for collaboration on the subject presented in this paper, for precious suggestions and very useful comments. 


\section{Appendix A: The total variation of opacity $\delta \kappa_{\mathrm{tot}}(r)$}

In order to calculate the total variation of opacity $\delta \kappa_{\text {tot }}(r)$ at a given radius $r$, we have to take into account that the perturbed sun has different temperature, density and chemical composition profiles with respect to SSM. We define:

$$
\delta \kappa^{\mathrm{tot}}(r)=\frac{\kappa\left(\rho(r), T(r), Y(r), Z_{\mathrm{i}}(r)\right)}{\bar{\kappa}\left(\bar{\rho}(r), \bar{T}(r), \bar{Y}(r), \bar{Z}_{\mathrm{i}}(r)\right)}-1
$$

and, by expanding the above equation to linear order, we obtain:

$$
\delta \kappa^{\mathrm{tot}}(r)=\kappa_{\mathrm{T}} \delta T(r)+\kappa_{\rho} \delta \rho(r)+\kappa_{\mathrm{Y}} \Delta Y(r)+\sum_{\mathrm{i}} \kappa_{\mathrm{i}} \delta Z_{\mathrm{i}}(r)+\delta \kappa_{\mathrm{I}}(r)
$$

where:

$$
\begin{array}{rlrl}
\kappa_{\mathrm{T}}(r) & =\left.\frac{\partial \ln \kappa}{\partial \ln T}\right|_{\mathrm{SSM}} & \kappa_{\rho}(r) & =\left.\frac{\partial \ln \kappa}{\partial \ln \rho}\right|_{\mathrm{SSM}} \\
\kappa_{Y}(r)=\left.\frac{\partial \ln \kappa}{\partial Y}\right|_{\mathrm{SSM}} & \kappa_{\mathrm{i}}(r)=\left.\frac{\partial \ln \kappa}{\partial \ln Z_{\mathrm{i}}}\right|_{\mathrm{SSM}}
\end{array}
$$

and the symbol $\left.\right|_{\mathrm{SSM}}$ indicates that we that we calculate the derivatives $\kappa_{j}(r)$ along the density, temperature and chemical composition profiles predicted by the SSM. The quantity $\delta \kappa_{\mathrm{I}}(r)$ represents the intrinsic opacity change and it is given by:

$$
\delta \kappa_{\mathrm{I}}(r)=\frac{\kappa\left(\bar{\rho}(r), \bar{T}(r), \bar{Y}(r), \bar{Z}_{\mathrm{i}}(r)\right)}{\bar{\kappa}\left(\bar{\rho}(r), \bar{T}(r), \bar{Y}(r), \bar{Z}_{\mathrm{i}}(r)\right)}-1
$$

By taking advantage of the equation of state of the stellar plasma, we can eliminate the density from Eq. (37). We use the relation:

$$
\delta \rho(r)=\delta P(r)-\delta T(r)-P_{Y}(r) \Delta Y(r)
$$

where $P_{Y}(r) \simeq-\partial \ln \mu / \partial Y \simeq-5 /[8-5 Y(r)]$ and $\mu$ represents the mean molecular weight, and we obtain:

$$
\delta \kappa^{\mathrm{tot}}(r)=\left(\kappa_{\mathrm{T}}-\kappa_{\rho}\right) \delta T(r)+\kappa_{\rho} \delta P(r)+\left(\kappa_{Y}-P_{Y} \kappa_{\rho}\right) \Delta Y(r)+\sum_{\mathrm{i}} \kappa_{\mathrm{i}} \delta Z_{\mathrm{i}}(r)+\delta \kappa_{\mathrm{I}}(r)
$$

In order to evaluate eq. 40 , we need to estimate the chemical composition of the perturbed sun, i.e. the quantities $\delta Z_{\mathrm{i}}(r)$ and $\Delta Y(r)$. By using the approximate method introduced in ref. [1] and reviewed in the next section, we can relate the chemical composition profiles to the modification of the photospheric heavy element admixture, to the present values of $\delta T(r)$ and $\delta P(r)$ and to the parameters $\Delta Y_{\text {ini }}$ and $\delta C$ which represent the absolute variation of the initial helium abundance and the fractional variation of the pressure at the bottom of the convective region, respectively. We obtain:

$$
\delta \kappa^{\mathrm{tot}}(r)=\kappa_{\mathrm{T}}^{\prime} \delta T(r)+\kappa_{\mathrm{P}}^{\prime} \delta P(r)+\kappa_{Y}^{\prime} \Delta Y_{\mathrm{ini}}+\kappa_{\mathrm{C}} \delta C+\left[\delta \kappa_{\mathrm{I}}(r)+\delta \kappa_{\mathrm{Z}}(r)\right]
$$

where $\delta \kappa_{\mathrm{Z}}(r)$ is the composition opacity change given by:

$$
\delta \kappa_{\mathrm{Z}}(r)=\sum_{i} \kappa_{\mathrm{i}} \delta z_{\mathrm{i}}
$$


where $\delta z_{\mathrm{i}}$ is the fractional variation of $z_{\mathrm{i}}=Z_{\mathrm{i}, \mathrm{b}} / X_{\mathrm{b}}$, while:

$$
\begin{aligned}
\kappa_{\mathrm{T}}^{\prime} & =\kappa_{\mathrm{T}}-\kappa_{\rho}\left(1+P_{Y} \xi_{\mathrm{T}}\right)-K_{Y} \xi_{\mathrm{T}} \\
\kappa_{\mathrm{P}}^{\prime} & =\kappa_{\rho}\left(1-P_{Y} \xi_{\mathrm{P}}\right)-K_{Y} \xi_{\mathrm{P}} \\
\kappa_{\mathrm{Y}} & =\left(\kappa_{Y}-\kappa_{\rho} P_{Y}\right) \xi_{Y}+Q_{\mathrm{Y}} \kappa_{\mathrm{Z}} \\
\kappa_{\mathrm{C}} & =Q_{\mathrm{C}} k_{\mathrm{Z}}
\end{aligned}
$$

with the coefficients $Q_{\mathrm{h}}$ and $\xi_{\mathrm{h}}$ defined in the next section. In the derivation of the above equation, we took into account that $\sum_{i} \kappa_{i}=\kappa_{\mathrm{Z}}$ where $\kappa_{\mathrm{Z}}=\partial \ln \bar{\kappa} / \partial \ln Z$ is the partial derivative of opacity with respect to the total metal abundance (i.e. calculated by rescaling all the heavy element abundances by a constant factor, so that the metal admixture remains fixed).

It is useful to define the opacity change $\delta \kappa(r)$ given by:

$$
\delta \kappa(r)=\delta \kappa_{\mathrm{I}}(r)+\delta \kappa_{\mathrm{Z}}(r)
$$

which groups together the contributions to $\delta \kappa^{\text {tot }}(r)$ which are directly related to the variation of the input parameters. While the other terms in eq. (41) represent derived quantities that are determined by solving the structure equations and/or by fitting the observed properties of the sun, the opacity change $\delta \kappa(r)$ can be varied, in principle, in arbitrary way.

\section{Appendix B: The chemical composition of the sun}

The chemical composition of the perturbed sun should be calculated by integrating the the perturbed structure and chemical-evolution equations starting from an ad-hoc chemical homogeneous ZAMS model. In ref.[1], we proposed a simplified approximate procedure that allows to estimate with sufficient accuracy the helium and metal abundances of the modified sun, without requiring to follow explicitly its time-evolution. We review this procedure and we extend it to take into account the effect of a variation of the photospheric composition.

In order to quantify the relevance of the different mechanisms determining the present composition of the sun, we express the helium and metal abundance according to:

$$
\begin{aligned}
& Y(r)=Y_{\text {ini }}\left[1+D_{Y}(r)\right]+Y_{\text {nuc }}(r) \\
& Z_{i}(r)=Z_{\mathrm{i}, \text { ini }}\left[1+D_{Z}(r)\right]
\end{aligned}
$$

Here, $Y_{\text {ini }}$ and $Z_{\mathrm{i} \text {,ini }}$ are the initial values for the abundances, the terms $D_{Y}(r)$ and $D_{Z}(r)$ describe the effects of elemental diffusion and $Y_{\text {nuc }}(r)$ represents the total amount of helium produced in the shell $r$ by nuclear processes. We note that we, implicitly, assumed that heavy elements have all the same diffusion velocity by introducing a common diffusion term $D_{Z}(r)$ for all metals.

We are interested in describing how the chemical composition is modified when we perturb the SSM. In the radiative core $\left(r \leq \bar{R}_{\mathrm{b}}\right)$, we neglect the effects produced by variations of the diffusion terms 10 and we write:

$$
\begin{aligned}
\Delta Y(r) & =\Delta Y_{\text {ini }}\left[1+\bar{D}_{Y}(r)\right]+\Delta Y_{\text {nuc }}(r) \\
\delta Z_{\mathrm{i}}(r) & =\delta Z_{\mathrm{i}, \text { ini }}
\end{aligned}
$$

\footnotetext{
${ }^{10}$ The diffusion terms $D_{Y}(r)$ and $D_{Z}(r)$ are at the few per cent level in the radiative region. Their variations are, thus, expected to produce very small effects on the solar composition.
} 
where $\Delta Y_{\text {nuc }}(r)$ is the absolute variation of the amount of helium produced by nuclear reactions. A better accuracy is required in the convective region, because the surface helium abundance $Y_{\mathrm{b}}$ is an observable quantity. We, thus, discuss explicitly the role of diffusion and we write:

$$
\begin{aligned}
\Delta Y_{\mathrm{b}} & =\left(1+\bar{D}_{Y, \mathrm{~b}}\right) \Delta Y_{\mathrm{ini}}+\bar{Y}_{\mathrm{ini}} \bar{D}_{Y, \mathrm{~b}} \delta D_{Y, \mathrm{~b}} \\
\delta Z_{\mathrm{i}, \mathrm{b}} & =\delta Z_{\mathrm{i}, \text { ini }}+\frac{\bar{D}_{Z, \mathrm{~b}}}{1+\bar{D}_{Z, \mathrm{~b}}} \delta D_{Z, \mathrm{~b}}
\end{aligned}
$$

where $\delta D_{Y, \mathrm{~b}}$ and $\delta D_{Z, \mathrm{~b}}$ are the fractional variations of the diffusion terms $D_{Y, \mathrm{~b}}$ and $D_{Z, \mathrm{~b}}$. The quantities $\Delta Y_{\mathrm{b}}$ and $\delta Z_{\mathrm{b}}$ are related among each other, since the metals-to-hydrogen ratios at the surface of the sun are observationally fixed. If we indicate with $z_{\mathrm{i}}=Z_{\mathrm{i}, \mathrm{b}} / X_{\mathrm{b}}$ the surface abundance of the $i$-element (rescaled to that of hydrogen), we obtain:

$$
\delta Z_{\mathrm{i}, \mathrm{b}}=-\frac{1}{1-\bar{Y}_{\mathrm{b}}} \Delta Y_{\mathrm{b}}+\delta z_{\mathrm{i}}
$$

where we considered that $X_{\mathrm{b}} \simeq 1-Y_{\mathrm{b}}$, while $\delta z_{\mathrm{i}}$ is defined by:

$$
\delta z_{\mathrm{i}}=\frac{\left(Z_{\mathrm{i}, \mathrm{b}} / X_{\mathrm{b}}\right)-\left(\bar{Z}_{\mathrm{i}, \mathrm{b}} / \bar{X}_{\mathrm{b}}\right)}{\left(\bar{Z}_{\mathrm{i}, \mathrm{b}} / \bar{X}_{\mathrm{b}}\right)}
$$

The above relation can be rewritten in terms of the initial helium and metal abundances, obtaining:

$$
\delta Z_{\mathrm{i}, \mathrm{ini}}=Q_{0} \Delta Y_{\mathrm{ini}}+Q_{1} \delta D_{Y, \mathrm{~b}}+Q_{2} \delta D_{Z_{\mathrm{b}}}+\delta z_{\mathrm{i}}
$$

The coefficients $Q_{i}$ have been calculated explicitly in ref.[1] and are given by $Q_{0}=-1.141, Q_{1}=0.041$ and $Q_{2}=+0.118$, respectively.

Up to this point, the derived relations have a general validity, since the only assumption implied by our analysis is that the heavy elements have all the same diffusion velocity (we take iron as representative for all metals). To complete our calculation, we have to estimate the term $\Delta Y_{\text {nuc }}(r)$ in eq. (45) and the quantities $\delta D_{Y, \mathrm{~b}}$ and $\delta D_{Z, \mathrm{~b}}$ in eqs.46, 49). We use the procedure adopted in the LSM approach, where we assumed that the helium produced by nuclear reactions scales proportionally to the energy generation coefficient (and, thus, the helium production rate) in the present sun, i.e:

$$
\Delta Y_{\text {nuc }}=\bar{Y}_{\text {nuc }}(r) \delta \epsilon^{\text {tot }}(r)
$$

where $\delta \epsilon^{\text {tot }}(r)$ is the fractional variation of the energy generation rate. The effect of elemental diffusion is modelled by assuming that the terms $D_{\mathrm{i}, \mathrm{b}}$ vary proportionally to the efficiency of diffusion in the present sun, obtaining (see sect.6.3 and appendix C of ref.[1]):

$$
\begin{aligned}
& \delta D_{Y, \mathrm{~b}}=\Pi_{Y} \delta T_{\mathrm{b}}+\Pi_{P} \delta P_{\mathrm{b}} \\
& \delta D_{Z, \mathrm{~b}}=\Pi_{Z} \delta T_{\mathrm{b}}+\Pi_{P} \delta P_{\mathrm{b}}
\end{aligned}
$$

where $\Pi_{Y}=2.05, \Pi_{Z}=2.73$ and $\Pi_{P}=-1.10$.

By following the calculations described in sect. 6.2 of [1], we obtain the following expression for the variation of the helium abundance in the radiative region:

$$
\Delta Y(r)=\xi_{Y}(r) \Delta Y_{\mathrm{ini}}+\xi_{T}(r) \delta T(r)+\xi_{P}(r) \delta P(r)
$$

The coefficients $\xi_{h}(R)$ are defined in eq.(31) of ref.[1] and are shown in their fig. 4. 
By taking into account relations (51) and by considering the conditions that hold at the bottom of the convective region (expressed in eq. (21) of [1]), we can estimate the variation of metal abundances in the radiative region, obtaining:

$$
\delta Z_{\mathrm{i}}(r)=\delta Z_{\mathrm{i}, \mathrm{ini}}=Q_{Y} \Delta Y_{\mathrm{ini}}+Q_{C} \delta C+\delta z_{\mathrm{i}}
$$

where $Q_{\mathrm{Y}}=-0.887, Q_{C}=-0.164$ and $\delta C=\delta P_{\mathrm{b}}$ represents the variation of pressure at the bottom of the convective envelope.

Finally, we can calculate the abundances in the convective region obtaining

$$
\begin{aligned}
\delta Y_{\mathrm{b}} & =A_{Y} \Delta Y_{\mathrm{ini}}+A_{C} \delta C \\
\delta Z_{\mathrm{i}, \mathrm{b}} & =B_{Y} \Delta Y_{\mathrm{ini}}+B_{C} \delta C+\delta z_{\mathrm{i}}
\end{aligned}
$$

where $A_{Y}=0.838, A_{C}=0.033, B_{Y}=-1.088$ and $B_{C}=-0.043$.

We remark that, while the quantities $\Delta Y_{\text {ini }}$ and $\delta C$ are parameters which are univocally determined by imposing the appropriate integration conditions (see next section), the quantities $\delta z_{\mathrm{i}}$ represent input parameters for solar model calculations.

\section{Appendix C: Linear Solar Models}

By expanding to linear order the structure equations of the present sun close to the SSM solution and by assuming that the variation of the chemical abundances of the sun can be estimated by the procedure outlined in the previous section, we obtain a linear system of ordinary differential equations that completely determine the physical and chemical properties of the "perturbed" sun (see [1] for details). Namely, we obtain:

$$
\begin{aligned}
\frac{d \delta m}{d r} & =\frac{1}{l_{m}}\left[\gamma_{P} \delta P+\gamma_{T} \delta T-\delta m+\gamma_{Y} \Delta Y_{\mathrm{ini}}\right] \\
\frac{d \delta P}{d r} & =\frac{1}{l_{P}}\left[\left(\gamma_{P}-1\right) \delta P+\gamma_{T} \delta T+\delta m+\gamma_{Y} \Delta Y_{\mathrm{ini}}\right] \\
\frac{d \delta l}{d r} & =\frac{1}{l_{l}}\left[\beta_{P}^{\prime} \delta P+\beta_{T}^{\prime} \delta T-\delta l+\beta_{Y}^{\prime} \Delta Y_{\mathrm{ini}}+\beta_{C}^{\prime} \delta C\right] \\
\frac{d \delta T}{d r} & =\frac{1}{l_{T}}\left[\alpha_{P}^{\prime} \delta P+\alpha_{T}^{\prime} \delta T+\delta l+\alpha_{Y}^{\prime} \Delta Y_{\mathrm{ini}}+\alpha_{C}^{\prime} \delta C+\delta \kappa\right]
\end{aligned}
$$

The coefficients $\gamma_{h}, \beta_{h}^{\prime}$ and $\alpha_{h}^{\prime}$ and the scale heights $l_{h} \equiv[d \ln (\bar{h}) / d r]^{-1}$ have been calculated in [1] and are shown in their fig. 1, fig. 5 and fig. 6 . The parameters $\Delta Y_{\text {ini }}$ and $\delta C$ represent the absolute variation of the initial helium abundance and the relative variation of pressure at the bottom of the convective envelope and can be univocally determined by imposing the appropriate integration conditions. At the center of the sun $(r=0)$ we have:

$$
\begin{aligned}
\delta m & =\gamma_{P, 0} \delta P_{0}+\gamma_{T, 0} \delta T_{0}+\gamma_{Y, 0} \Delta Y_{\mathrm{ini}} & & \delta P=\delta P_{0} \\
\delta l & =\beta_{P, 0}^{\prime} \delta P_{0}+\beta_{T, 0}^{\prime} \delta T_{0}+\beta_{Y, 0}^{\prime} \Delta Y_{\mathrm{ini}}+\beta_{C, 0}^{\prime} \delta C & \delta T & =\delta T_{0}
\end{aligned}
$$

where the subscript " 0 " indicates that a given quantity is evaluated at $r=0$. At the bottom of the convective envelope $\left(r=\bar{R}_{\mathrm{b}}\right)$, we have instead:

$$
\begin{aligned}
\delta m & =-\bar{m}_{\text {conv }} \delta C & & \delta P=\delta C \\
\delta l & =0 & & \delta T=A_{Y}^{\prime} \Delta Y_{\text {ini }}+A_{C}^{\prime} \delta C
\end{aligned}
$$


where $A_{Y}^{\prime}=0.626$ and $A_{C}^{\prime}=0.025$ and $\bar{m}_{\text {conv }}=\bar{M}_{\text {conv }} / M_{\odot}=0.0192$ is the fraction of solar mass contained in the convective region.

The term $\delta \kappa(r)$ contains the contributions to the modification of the opacity profile of the sun that are directly related to the variation of the input parameters. It is given by:

$$
\delta \kappa(r)=\delta \kappa_{\mathrm{I}}(r)+\delta \kappa_{\mathrm{Z}}(r)
$$

where $\delta \kappa_{\mathrm{I}}(r)$ and $\delta \kappa_{\mathrm{Z}}(r)$ are the intrinsic and composition opacity changes, defined in eqs.(3) and (5) respectively. It represents the source term that drives the modification of the solar properties an that can be bounded by observational data. 


\section{References}

[1] F. L. Villante and B. Ricci, Astrophys. J. 714 (2010) 944 [arXiv:0912.4696 [astro-ph.SR]].

[2] M. Asplund, N. Grevesse and A.J. Sauval, Astronomical Society of the Pacific Conference Series 336 (2005) 25, T.G. Barnes and F.N. Bash editors.

[3] M. Asplund, N. Grevesse, A.J. Sauval and P. Scott, Ann. Rev. Astr. Astroph. 47 (2009) 481.

[4] E. Caffau, H. G. Ludwig, M. Steffen, B. Freytag and P. Bonifacio, arXiv:1003.1190 [astro-ph.SR].

[5] N. Grevesse and A.J. Sauval, Space Science Rev. 85 (1998) 161.

[6] J.N. Bahcall et al. Astrophys.J. 618 (2005) 1049.

[7] S. Basu and H. M. Antia, Phys. Rept. 457 (2008) 217

[8] S. C. Tripathy and J. Christensen-Dalsgaard, Astronomy and Astrophysics, 337 (1998) 579

[9] F. L. Villante, J. Phys. Conf. Ser. 203 (2010) 012084 [arXiv:1001.2510 [astro-ph.SR]].

[10] A. Bottino, G. Fiorentini, N. Fornengo, B. Ricci, S. Scopel and F. L. Villante, Phys. Rev. D 66 (2002) 053005 arXiv:hep-ph/0206211].

[11] A. Chieffo and O. Straniero, ApJ Suppl. 17 (1989) 47; F. Ciacio, S. Degl'Innocenti and B. Ricci, Astr. Astroph. Suppl. 123 (1997) 449

[12] A. M. Serenelli, arXiv:0910.3690 [astro-ph.SR].

[13] F. L. Villante, talk presented at the he eleventh international conference on "Topics in Astroparticle and Underground Physics" (TAUP 2009), http://taup2009.lngs.infn.it/

[14] M. T. Frandsen and S. Sarkar, arXiv:1003.4505 [hep-ph].

[15] M. Taoso, F. Iocco, G. Meynet, G. Bertone and P. Eggenberger, arXiv:1005.5711 [astro-ph.CO].

[16] Q. R. Ahmad et al. [SNO Collaboration], Phys. Rev. Lett. 89 (2002) 011301 arXiv:nuclex/0204008.

B. Aharmim et al. [SNO Collaboration], Phys. Rev. C 72 (2005) 055502 arXiv:nucl-ex/0502021].

B. Aharmim et al. [SNO Collaboration], Phys. Rev. Lett. 101, 111301 (2008) arXiv:0806.0989 [nucl-ex]].

[17] C. Arpesella et al. [The Borexino Collaboration], Phys. Rev. Lett. 101, 091302 (2008) arXiv:0805.3843 [astro-ph]].

[18] C. Pena-Garay and A. Serenelli, arXiv:0811.2424 [astro-ph]. 